\title{
Ionospheric Weather Forecasting on the Horizon
}

In 1901, Guglielmo Marconi successfully transmitted radio signals across the Atlantic Ocean, and shortly thereafter the existence of the ionosphere was clearly established. Since its discovery, the ionosphere has been shown to display both a background state (climatology) and a disturbed state (weather). Ionospheric climatology has been successfully modeled, but ionospheric weather has been much more difficult to model because the ionosphere can vary significantly on an hour-by-hour basis. Unfortunately, ionospheric weather can have detrimental effects on several human activities and systems, including high-frequency communications, over-the-horizon radars, and survey and navigation systems using Global Positioning System (GPS) satellites.

In an effort to mitigate the adverse effects of the ionosphere on military and civilian operations, specification and forecast models are being developed that use state-of-the-art data assimilation techniques. Utah State University (USU) has recently developed two data assimilation models for the ionosphere as part of the USU Global Assimilation of Ionospheric Measurements (GAIM) program. One of these models is currently being implemented at the Air Force Weather Agency for operational use. The USU-GAIM models are also being used for scientific studies, and this should lead to a dramatic advance in our understanding of ionospheric physics; similar to what occurred in meteorology and oceanography after the introduction of data assimilation models in those fields.

As shown by the meteorologists and oceanographers, the most reliable weather models are the physics-based, data-driven models that use Kalman filter or other data assimilation techniques. Since the state of a medium (ocean, lower atmosphere, ionosphere) is driven by complex and frequently nonlinear internal and external processes, it is not possible to accurately specify all of the drivers and initial conditions of the medium. Therefore physics-based models alone cannot provide reliable specifications and forecasts. Data assimilation techniques were introduced to augment the physics-based models.

These two physics-based data assimilation models of the type used in meteorology and oceanography were developed as part of a Department of Defense Multidisciplinary University Research Initiative program. Both models are capable of assimilating data from a variety of data sources, including in situ electron densities from satellites, bottomside electron density profiles from ionosondes, total electron content (TEC) measurements between ground receivers and the GPS satellites, occultation data from satellite constellations, and ultraviolet emissions from the ionosphere measured by satellites (Figure 1). 


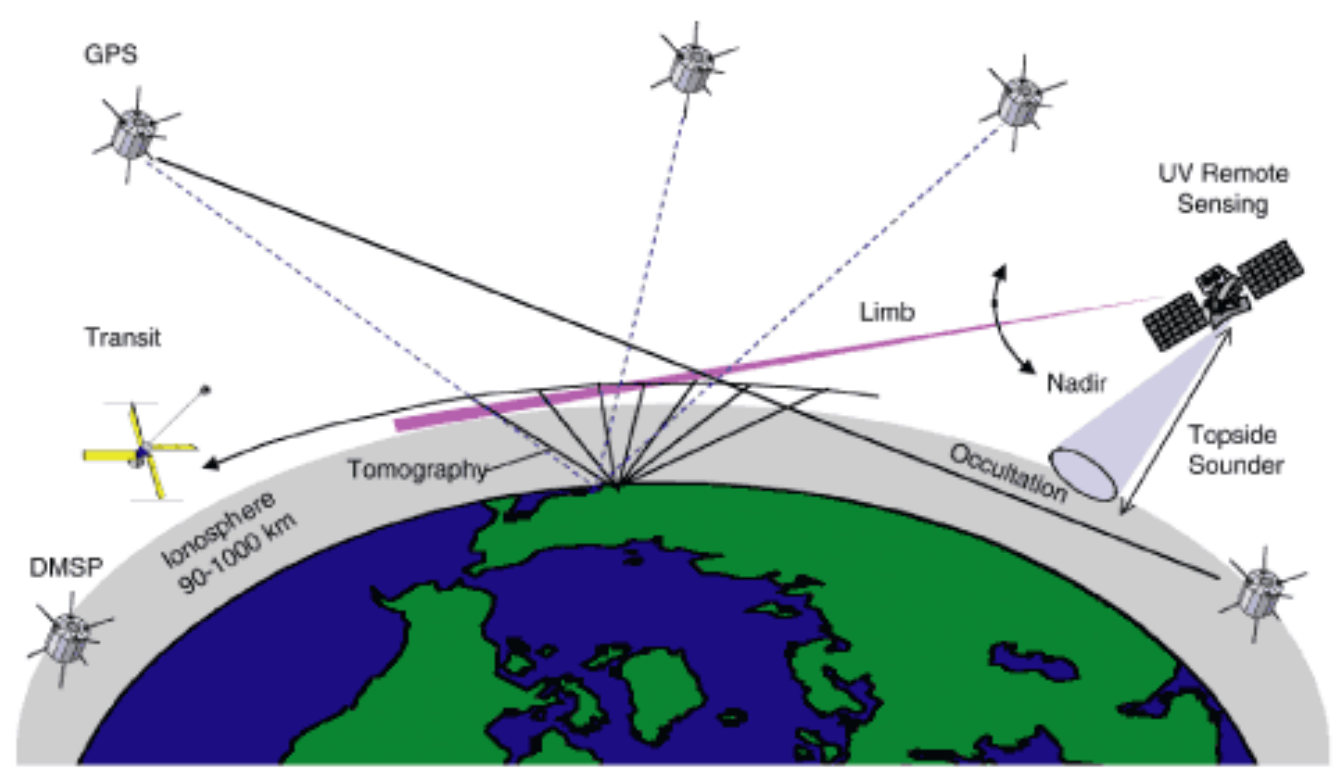

Figure 1. The Utah State University Global Assimilation of Ionospheric Measurements (USU GAIM) models incorporate a variety of data sources to augment the physics-based models that by themselves cannot provide reliable specifications and forecasts.

\section{New Tools for Science and Operational Forecasting}

The USU-GAIM models have been selected for operational use at the Air Force Weather Agency in Omaha, Nebraska. They are also running at the Naval Research Laboratory in Washington, D. C., and the Air Force Research Laboratory in Bedford, Massachusetts. Discussions have also been initiated to implement the USUGAIM models at the Community Coordinated Modeling Center at NASA Goddard Space Flight Center. The models are being considered for operational use at the NOAA National Weather Service, which provides space weather products for civilian customers.

These new models will provide a significant improvement in Air Force ionospheric specifications and forecasts, which are currently based on the parameterized real-time ionospheric specification model (PRISM). PRISM is based on a limited number of global data sets calculated from an old version of the ionospheric forecast model (IFM). The resulting three-dimensional electron density fields are locally adjusted to agree with measurements of vertical total electron content, in situ satellite data, and ionosonde parameters (F-region peak height and density). The net result is that PRISM produces localized density peaks and holes at the coordinates of the measurements, and these features are then scaled back to the IFM background electron density field over some predetermined horizontal scale length.

The USU-GAIM models, on the other hand, are based on an updated IFM, state-of-the-art Kalman filter data assimilation techniques, and correlation lengths determined by the physics. They can also assimilate data from an arbitrary number of sources associated with several different data types.

\section{Gauss-Markov Data Assimilation Model}

The most mature of the two USU models is the Gauss-Markov data assimilation model, which uses a physicsbased model of the ionosphere and a Kalman filter as a basis for assimilating a diverse set of real-time (or near real-time) measurements. The physics-based model is the IFM, which is global and covers the E region, $\mathrm{F}$ region, and topside from 90 to $1400 \mathrm{~km}$. It takes account of electrons and five ion species $(\mathrm{NO}+, \mathrm{O} 2+, \mathrm{N} 2+$, $\mathrm{O}+, \mathrm{H}+)$, electron and ion temperatures, and plasma drifts both parallel and perpendicular to the geomagnetic field. 
Some of the ionospheric drivers in the IFM (electric fields, particle precipitation, neutral winds), however, are provided by empirical models, which are varied in time according to changing geophysical conditions such as season, solar cycle, and magnetic activity. The Gauss-Markov Kalman model assimilates bottomside Ne profiles from a variable number of ionosondes, slant TEC from a variable number of ground GPS/TEC stations, in situ Ne from four Defense Meteorological Satellite Program spacecraft, and line-of-sight ultraviolet emissions measured by satellites.

With the Gauss-Markov model, the ionospheric densities obtained from the IFM constitute a background ionospheric density field on which perturbations are superimposed based on the available data sources and their errors. The density perturbations and the associated errors evolve over time via a statistical Gauss-Markov process. The model has both regional and global capabilities; the output is a three-dimensional distribution of electron densities at user-specified times.

The Gauss-Markov model was delivered to the Air Force Weather Agency in July 2004 and is currently being transitioned for operational use. It was also delivered to the Naval Research Laboratory and the Air Force Research Laboratory for validation purposes. This model has been running continuously and autonomously at USU in an operational, near-real-time setting since January 2003. In this operational setting, the Gauss-Markov model automatically collects data on the World Wide Web (from more than 200 ground GPS/TEC receivers and 20 digisondes), quality controls the data, assimilates the data, and produces global electron density distributions at 15-min intervals. During both quiet and disturbed periods, the data are visually compared with data that are not assimilated, including TOPEX and JASON TEC satellite data and ionosonde data taken at the Bear Lake Observatory in Garden City, Utah.

A snapshot of the output from the operational Gauss-Markov data assimilation model is shown in Figure 2. As noted above, slant TEC data from more than 200 ground receivers and bottomside electron density profiles from 20 ionosondes are assimilated at a 15-min interval into this model. Snapshots like these, as well as other outputs, are produced continuously and automatically throughout the year by the operational model.
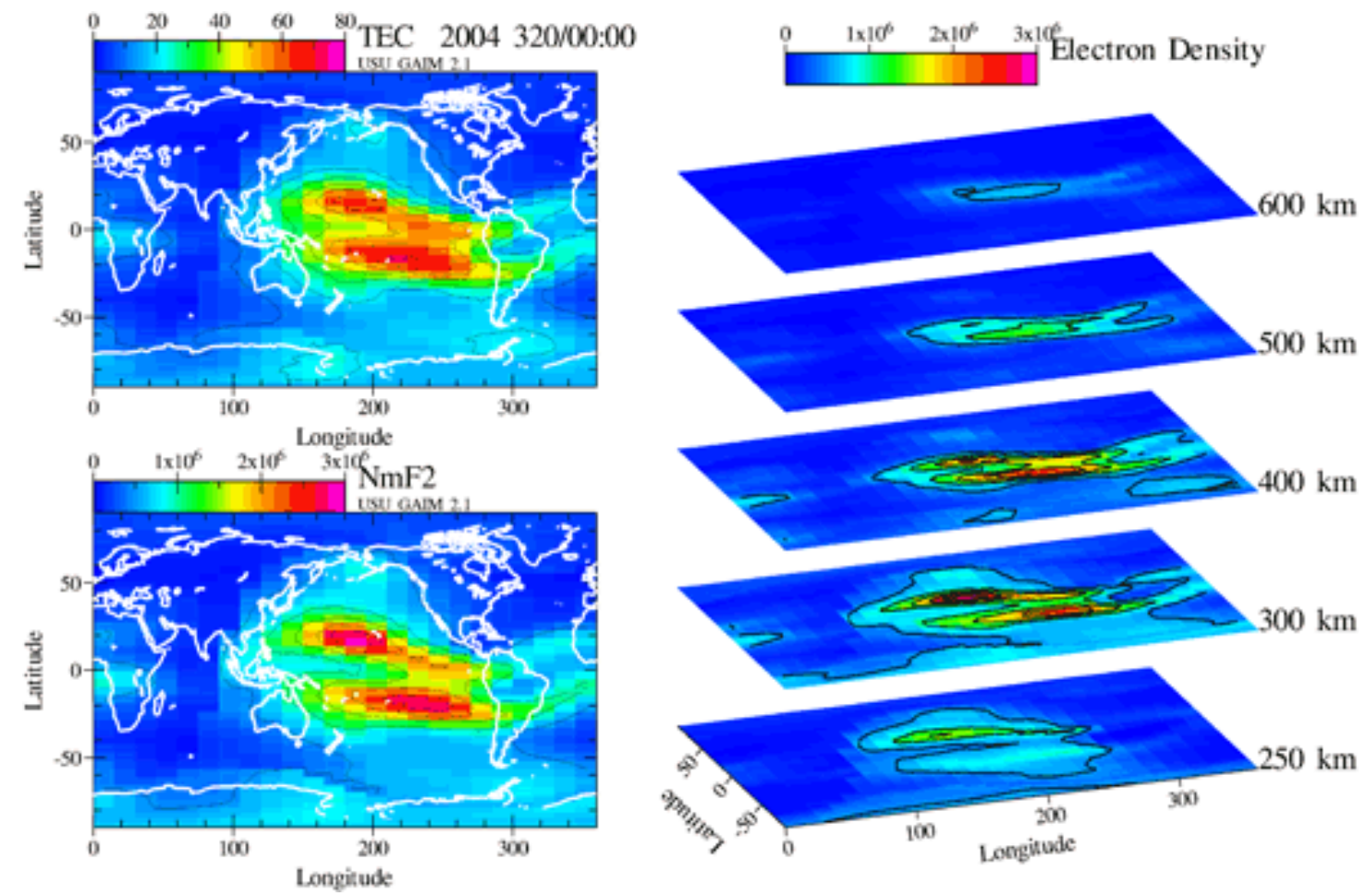

Figure 2. One of the standard outputs of the operational version of the Gauss-Markov data assimilation model shows (top left) a global distribution of the vertical TEC obtained by integrating through the three-dimensional electron density distribution from 90 to $1400 \mathrm{~km}$, (bottom left) the peak electron density in the F-region $(\mathrm{NmF} 2)$, and (right) the electron density variation at selected altitudes. Clearly visible in this output for day 320 in 2004 (0000 UT) are the well-known equatorial anomaly peaks that occur on both sides of the magnetic equator. 
The Gauss-Markov model has also been used for scientific studies. Figure 3 shows a snapshot from a regional run of the model conducted in order to study the large geomagnetic storm on 20-21 November 2003. Note the region of storm-enhanced density that extends from Florida to the Great Lakes. Ground GPS/TEC measurements from more than 300 GPS receivers covering the United States and Canada were used in the model. More than 2000 slant TEC values were assimilated every 15 min. Bottomside electron density profiles from ionosondes at Dyess and Eglin were also assimilated.

\section{The Next Step: A Full-Physics Data Assimilation Model}

Still under development at USU is a full-physics, Kalman filter data assimilation model that is more sophisticated than the Gauss-Markov model. This model is scheduled to be delivered to the Air Force Weather Agency in 2006. The model is based on a new physicsbased model of the ionosphere-plasmasphere-polar wind system, covering the altitude range from 90 to 20,000 $\mathrm{km}$. The physics-based model is state of the art and includes six ion species $(\mathrm{NO}+, \mathrm{O} 2+, \mathrm{N} 2+, \mathrm{O}+, \mathrm{He}+$, $\mathrm{H}+$ ), ion and electron temperatures, and plasma drifts parallel and perpendicular to the geomagnetic field.

The full-physics data assimilation model rigorously evolves the three-dimensional electron density field and its associated errors using the full-physical model. During severe space weather this is an advantage over the Gauss-Markov model, where the errors evolve statistically. Another advantage is that the full-physics data assimilation model also provides global distributions of the self-consistent drivers of the ionosphere, including the neutral winds and composition, the electric fields, and the particle precipitation. However, as with the Gauss-Markov model, the full-physics model uses a Kalman filter for the data assimilation and can incorporate the same data sources.

These new ionospheric data assimilation models will continuously track the global electron density variations throughout the year, and the results will be available for commercial and military products as well as for scientific studies. Consequently, during this decade, data assimilation models of the ionosphere should lead to a major advance of the field similar to the advances in meteorology and oceanography after physics-based data assimilation models were introduced in those fields.

\section{Robert W. Schunk is a professor of physics and director of the Center for Atmospheric and Space Sciences at Utah State University in Logan, Utah.}

\section{Ludger Scherliess is a research scientist in the Center for Atmospheric and Space Sciences at Utah State University in Logan, Utah.}
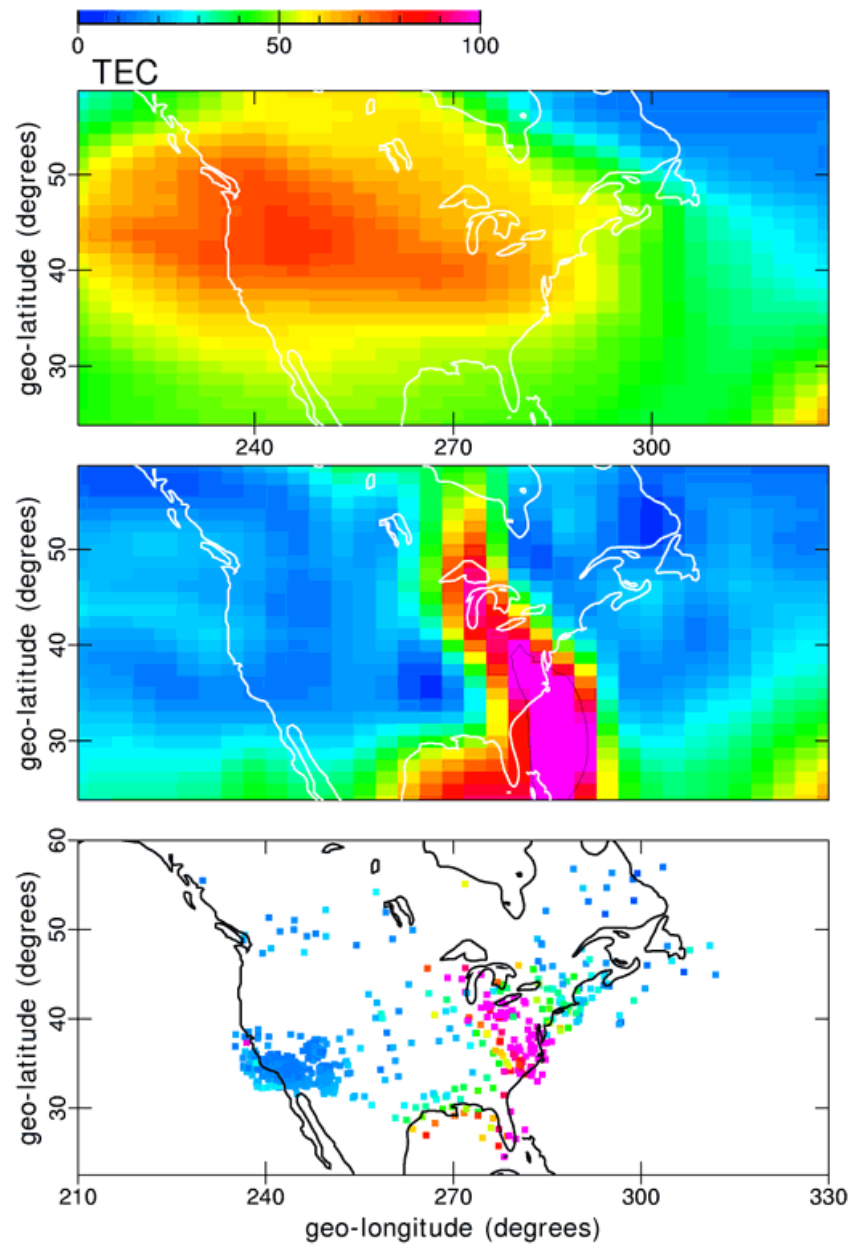

2003/324 20:00

Figure 3. A snapshot of a regional run of the Gauss-Markov data assimilation model for the geomagnetic storm in November 2003 shows (top) the output from the physicsbased ionosphere forecast model with no data assimilation, (bottom) the measured total electron content plotted at the $350-\mathrm{km}$ pierce point (slant TEC converted to the vertical), and (middle) the Gauss-Markov reconstruction, where the slant TECs are assimilated in the ionosphere forecast model. The snapshot is for day 324 in 2003 (2000 UT). 
Sciences at Utah State University in Logan, Utah.

Donald C. Thompson is a research scientist in the Center for Atmospheric and Space Sciences at Utah State University in Logan, Utah.

Lie Zhu is a research scientist in the Department of Physics and the Center for Atmospheric and Space Sciences at Utah State University in Logan, Utah.

Citation: Schunk, R., L. Scherliess, J. J. Sojka, D. C. Thompson, and L. Zhu (2005), Ionospheric Weather Forecasting on the Horizon, Space Weather, 3, S08007, doi:10.1029/2004SW000138.

Copyright 2005 by the American Geophysical Union. 